\title{
Discovery of new dwarf galaxies around NGC4631 with Subaru/Hyper Suprime-Cam
}

\author{
Mikito Tanaka ${ }^{1}$, Masashi Chiba ${ }^{2}$ and Yutaka Komiyama ${ }^{3}$ \\ ${ }^{1}$ Frontier Research Institute for Interdisciplinary Sciences, Tohoku University, Sendai, Japan \\ email: mikito@astr.tohoku.ac.jp \\ ${ }^{2}$ Astronomical Institute, Tohoku University, Sendai, Japan \\ ${ }^{3}$ Subaru Telescope, National Astronomical Observatory of Japan
}

\begin{abstract}
We have observed on-going interacting galaxies (NGC4631 and NGC4656) using Subaru/Hyper Suprime-Cam and reduced the data using HSC pipeline and conducted photometry based on DAOphot. Then, we have detected 8 new dwarf galaxy candidates in the outer region of NGC4631 and confirmed the three candidates previously reported by Karachentsev et al. 2014. The 3 or 4 candidates detected in this study may be a star-forming dwarf irregular galaxy and the other 7 candidates may be an old dwarf spheroidal galaxy based on these stellar populations. It looks like that the effective radius - absolute magnitude relation of dwarf galaxies in NGC4631 group is similar to the relation of the Local Group and the other galaxy systems.
\end{abstract}

Keywords. galaxies: dwarf - galaxies: stellar content - galaxies: halo - galaxies: individual (NGC 4631, NGC 4656)

\section{Introduction}

Current large telescopes enable us to investigate resolved stellar populations of halos of more distant galaxies than the Milky Way (MW) and the Andromeda galaxy. Halos of several galaxies of different type, from ellipticals to late spirals, were studied. For example, Tanaka et al. (2011) carried out imaging observations of the halo of NGC55, which is less massive tham MW and M31, using Subaru/Suprime-Cam, and identified some overdense stellar structures and low-metallicity stellar populations in its outskirts. The presence of halo substructures and a heated thick disk with the abnormally large scale height suggests that NGC55 has hierarchically formed in the same way as M31 and MW. Such a recent discovery of the diversity of halo structures whose detailed physical parameters such as metallicity and luminosity of halos depend on morphology of hostgalaxy suggests the possibility that formation scenario of galactic halos is also depending on morphology of host-galaxy.

\section{This work}

In order to investigate structure and state of an outer region of on-going interacting galaxy we have observed a nearby on-going interacting galaxy group mainly consisting of NGC4631 and NGC4656 using the Hyper Suprime-Cam (HSC) on the Subaru telescope. The exposure times were $48 \mathrm{~min}$ in i-band and $36 \mathrm{~min}$ in g-band. The seeing was over 1". In this study, we adopt the distance of $7.1 \mathrm{Mpc}$ for this group from Tikhonov et al. (2006).

We have basically reduced our HSC image using HSC pipeline ver.3, and we have performed photometric calibration and astrometry based on SDSS DR7 data covering whole regions of our HSC field-of-view. We have newly discovered 8 dwarf galaxy candidates 


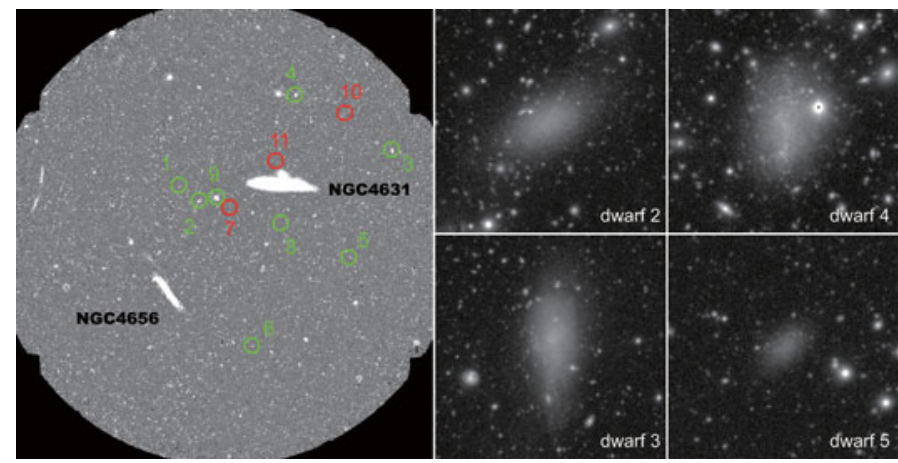

Figure 1. Left: the spatial distribution of dwarf galaxies in NGC4631 group. Right: 4 representative samples of all dwarf galaxy candidates we have photometrically detected.

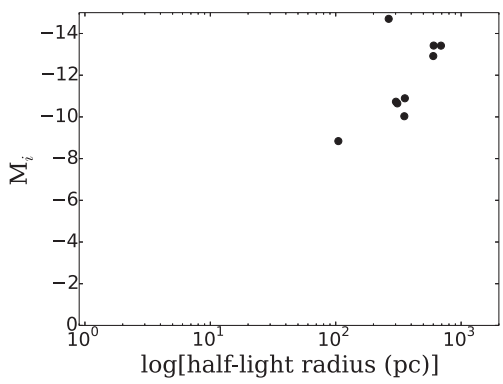

Figure 2. The effective radius vs. absolute magnitude relation of NGC4631's dwarf galaxies. Because of the low surface brightness of Dwarfs 10 and 11 close proximity to a bright star of Dwarf 7, we cannot determine physical parameters of them in this shallow observation.

(green circles in Fig. 1) by visual inspection. In addition, we have confirmed the 3 dwarf candidates which Karachentsev et al. (2014) found in their previous study (three red circles in Fig. 1). Apparently, these dwarf galaxies are isotropically distributed around NGC4631 (not NGC4656) in contrast to dwarf galaxies of MW and M31 distributed in a thin plane (e.g., Pawlowski et al. 2012 and Ibata et al. 2013).

On the other hand, from our HSC i-band image, we determine a position of dwarf candidates with SExtractor, and conduct surface photometry using PyRAF/ELLIPSE package. Furthermore, we measure physical properties such as position angle, ellipticity, Sersic indices and effective radius. Fig. 2 shows the effective radius vs. absolute magnitude relation for dwarf galaxies in NGC4631 group. The relation suggests that these dwarf galaxies with brighter total luminosity probably tend to be more spatially extended. Furthermore, the relation is similar to the one of the Local Group and the other galaxy systems (e.g., Gilmore et al. 2007), implying that the dwarf galaxy system of the NGC4631 group may have formed through similar processes as the Local Group.

\section{References}

Gilmore et al. 2007, ApJ, 663, 948

Ibata et al. 2013, Nature, 493, 62

Pawlowski et al. 2012, MNRAS, 423, 1109

Tanaka et al. 2011, ApJ, 738, 150

Tikhonov et al. 2006, astro-ph/0603457

Karachentsev et al. 2014, astro-ph/1401.2719 